Instituto Internacional de Investigación y Desarrollo Tecnológico Educativo INDTEC, C.A.

DOI: https://doi.org/10.29394/Scientific.issn.2542-2987.2020.5.16.15.290-304

OAI-PMH: http://www.indteca.com/ojs/index.php/Revista Scientific/oai

Ensayo Original / Original Essay

\title{
X-Y. Ahora vienen los Z: Una Generación de nuevos Ziudadanos
}

Autores: David Ismael Delgado Velesaca Universidad Católica de Cuenca, UCACUE david.delgadov@outlook.com Cuenca, Ecuador https://orcid.org/0000-0002-1498-1626

Diego Heriberto Urgilés Quintuña Universidad Católica de Cuenca, UCACUE diegourgilesg@hotmail.com Cuenca, Ecuador https://orcid.org/0000-0002-6837-9438

Patricia Katherine Vega Feijoó Universidad Católica de Cuenca, UCACUE patricia.vegaf@outlook.es Cuenca, Ecuador https://orcid.org/0000-0001-5485-9775

\section{Resumen}

El incremento de recursos tecnológicos en los centros educativos se ha convertido en la tendencia actual, su propósito, mejorar los procesos de enseñanza-aprendizaje. Los denominados Generación $Z$ han nacido en el auge digital, para ellos la tecnología es parte de su vida, sin embargo, la simple presencia de equipamiento o las facilidades para la interconexión no bastan para el desarrollo de competencias digitales, se requiere, además de lo anterior, el compromiso de todos los actores educativos, en especial del docente. El propósito del presente ensayo es analizar el nivel de competencias digitales que deben poseer los estudiantes denominados Generación Z, estudios realizados evidencian la necesidad de incorporar metodologías de enseñanza con la finalidad de dar respuesta a necesidades futuras superando esquemas tradicionales.

Palabras clave: competencias del docente; enseñanza multimedia; estudiante; tecnología educacional.

\section{Cómo citar este ensayo:}

Delgado, D., Urgilés, D., \& Vega, P. (2020). X-Y. Ahora vienen los Z: Una Generación de nuevos Ziudadanos. Revista Scientific, 5(16), 290-304, e-ISSN: 2542-2987. Recuperado de: https://doi.org/10.29394/Scientific.issn.2542-2987.2020.5.16.15.290-304

Fecha de Recepción: $11-12-2019$
Fecha de Aceptación: 23-03-2020
Fecha de Publicación: 05-05-2020 
Instituto Internacional de Investigación y Desarrollo Tecnológico Educativo INDTEC, C.A.

DOI: https://doi.org/10.29394/Scientific.issn.2542-2987.2020.5.16.15.290-304

OAI-PMH: http://www.indteca.com/ojs/index.php/Revista_Scientific/oai

Ensayo Original / Original Essay

\title{
X-Y. Now come the Z: A Generation of new Zitizens
}

\begin{abstract}
The increase of technological resources in educational centers has become the current trend, its purpose, to improve teaching-learning processes. The so-called Generation $Z$ have been born in the digital boom, for them technology is part of their life, however, the mere presence of equipment or the facilities for interconnection are not enough for the development of digital skills, it is required, in addition to what above, the commitment of all educational actors, especially the teacher. The purpose of this essay is to analyze the level of digital competences that students called Generation Z must possess, studies conducted show the need to incorporate teaching methodologies in order to respond to future needs by overcoming traditional schemes.
\end{abstract}

Keywords: teacher qualifications; multimedia instruction; student; educational technology.

How to cite this essay:

Delgado, D., Urgilés, D., \& Vega, P. (2020). X-Y. Now come the Z: A Generation of new Zitizens. Revista Scientific, 5(16), 290-304, e-ISSN: 2542-2987. Recovered from: https://doi.org/10.29394/Scientific.issn.2542-2987.2020.5.16.15.290-304
Date Received:

11-12-2019
Date Acceptance:

23-03-2020
Date Publication:

05-05-2020 


\section{Introducción}

En la actualidad es innegable la influencia que ejerce la tecnología y su uso dentro de los contextos socioculturales, la sociedad del conocimiento ha ocasionado enormes avances que saltan a la vista en cada aspecto del diario vivir. La educación no puede estar exenta de ello, cuando se señala la escuela, se refiere a todo el componente educativo, infraestructura, autoridades y docentes forman parte de este conglomerado que poseen la misión y la obligación de responder a la pregunta ¿Cómo enfrentar este nuevo reto educativo con generaciones que nacieron y crecieron en el boom tecnológico?

Como indican Pérez-Escoda, Castro-Zubizarreta y Fandos-Igado (2016a): los estudiantes de esta generación, son aquellos que desde el momento de su nacimiento han estado enmarcados en un mundo lleno de instrumentos y modelos tecnológicos, varios autores han intentado definir dicha generación con nombres tales como Generación Google, Generación V (Virtual), Generación Silenciosa y también como Generación Z.

Resulta oportuno marcar, que si se analiza la Generación $Z$ desde una mirada socio-cognitiva, poseen rasgos característicos que distan mucho de generaciones estudiantiles anteriores, dentro de esos engranajes característicos, Pérez-Escoda, Castro-Zubizarreta y Fandos-Igado (2016b): enumeran la capacidad de rápida respuesta y de interacciones continuas, la concepción de uso competente en Tics (colocando a la tecnología como su forma de aprendizaje independiente), su fulminante desenvolvimiento dentro de contextos digitales y su facilidad de resolución de varias tareas, fenómeno denominado multitasking.

Para Camacho (2018a): la Generación Z se desarrolla en un entorno creativo digital, trabajan en sinergia con el objeto de evolucionar del paradigma tradicional de educación y llegar al conectivismo. Los alumnos nativos digitales actúan de manera diferente que sus maestros migrantes digitales, por el mismo hecho de haber crecido en un ambiente tecnológico. 
Los requisitos para convertirse en un ciudadano digital e ingresar a la

Sociedad de la Información y Comunicación (SIC), están generando transformaciones en el campo educativo. Dadas las condiciones que anteceden, la educación tiene el desafío, por no decir la obligación, de dar respuestas que realmente aporten a los procesos educativos, no sobreestimando competencias estudiantiles, sino aportando participativa y críticamente tanto en el consumo como en la difusión de contenidos digitales.

\section{Educación 3.0: desarrollando competencias digitales}

El ser humano en su afán de controlar las cosas que lo rodean, ha optado siempre por clasificar y designar nombres, así nace la Generación Z, esta clasificación, hace referencia a los nacidos a finales del Siglo XX y principios del Siglo XXI, la característica principal de este grupo generacional es su facilidad para usar y desenvolverse en medios digitales, la tecnología es parte de su vida diaria y no conciben la idea de prácticas alejadas de dicha realidad.

En palabras de Pérez-Escoda, Castro-Zubizarreta y Fandos-Igado (2016c): los denominados $Z$, no se direccionan en algo específico, más bien buscan varios frentes a los cuales reaccionar, de ahí que se insista en el término "multitasking" o "multitarea" para definirlos, sin embargo esta capacidad de reacción muchas veces disminuye en el plano educativo ya que para ellos la actividad pedagógica es monótona y no se desarrolla frente a modalidades de tecnología que motiven su aprendizaje y muchos menos les ofrezcan la interacción que requieren, de ahí que la brecha entre los inmigrantes digitales (docentes) y la Generación Z (estudiantes) sea cada vez más significativa.

Desde el punto de vista de Ortega (2017): existen numerosos calificativos para esta generación entre ellas se destaca: la irreverencia; inmediatez; inclusión; e incertidumbre. Irreverentes, porque no tienen miedo 
a pensar diferente; Inmediatez, en la Web porque consiguen todo lo que buscan; Inclusivos, debido a que trabajan en comunidad virtual; e Incertidumbre, en el mundo en que nacieron porque todo cambia y nada es estable.

Para afrontar dicho reto, uno de los componentes más importantes es el docente, de acuerdo con Fernández-Cruz y Fernández-Díaz (2016a): éstos no poseen las competencias mínimas para enfrentar los procesos actuales que demanda esta nueva generación, esto representa una preocupación ya que dichos estudiantes no son preparados para contextos digitales donde sea imperativo el uso de competencias en busca de dar respuestas a necesidades futuras.

El Ministerio de Educación (MECD, 2013), citado por Fernández-Cruz y Fernández-Díaz (2016b): menciona que en España, de los 664 profesores existentes, solo 325 se ubican en primaria como en secundaria, el $59,2 \%$ son mayores de 40 años, teniendo en cuenta la terminología generacional por año de nacimiento, se puede determinar que el $40 \%$ del componente docente son parte de la Generación Y, el $30 \%$ son incluidos en el grupo de los Baby Boomers o primera generación. Lo que resulta un problema generacional inminente si se toma en cuenta datos estadísticos que otorga el Ministerio de Educación (MECD, 2014), citado por Fernández-Cruz y Fernández-Díaz (2016c): en el cual se establece que existen 8.801 .972 estudiantes que son escolarizados y que son parte de esta Generación Z.

Actualmente los sistemas educativos cuentan con profesionales de diversas edades, la gran mayoría pertenecientes a los denominados Baby Bommers y Generación X, como se puede apreciar en la tabla 1, las cuales se caracterizaban por un uso incipiente de rudimentarios inventos tecnológicos, el mundo en el cual se desarrollaban inició la exploración de tecnología básica la cual contribuyó a los avances futuros. Posteriormente surge la Generación $\mathrm{Y}$, inmersa en trasformaciones rápidas con la posibilidad de conexiones 
comunitarias, nacen las comunicaciones globales gracias a los correos electrónicos y mensajes de texto. Más recientemente surgen la Generación Z y Alpha, conocidos como nativos digitales para ellos la tecnología facilita su conexión con la realidad globalizada, explorando, creando e innovando más allá de la web 2.0.

Tabla 1. Clasificación generacional según el año de nacimiento.

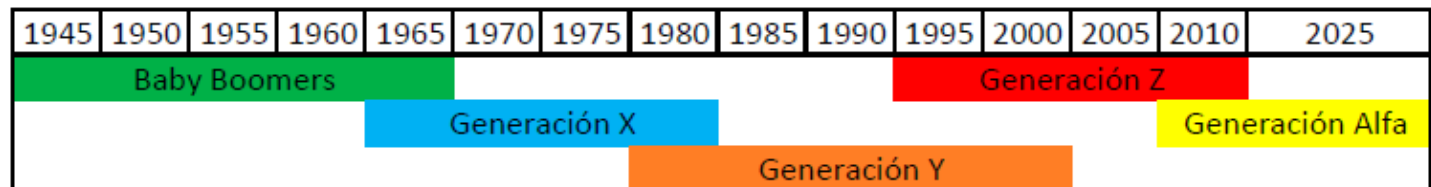

Fuente: Fernández-Cruz y Fernández-Díaz (2016).

El ejemplo anterior descrito evidencia una brecha entre profesorestudiante, lo que hace repensar cuán capacitado está el docente para atender dichas necesidades educativas y adaptarse a nuevas habilidades culturales y sociales que utilizan como medio las tecnologías, es por lo que la profesionalización docente debe incluir planes de capacitación permanentes que reduzcan la distancia de conocimientos existente con esta generación, la Z.

Conforme a los razonamientos que se han venido realizando, conviene enfocarse en analizar el desarrollo de competencias en espacios educativos, al respecto, el Ministerio de Educación, Cultura y Deporte (2015a); y Silva (2018a): manifiestan que las competencias son aquellas capacidades superiores que reflejan un "saber hacer", el cual condensa y vincula las destrezas y habilidades desarrolladas previamente, para aplicarlas en situaciones de un contexto determinado, de igual forma, se considera a la competencia como capacidades esenciales en el ser humano para su desarrollo personal frente a contextos globales de inclusión social.

En consonancia con Pérez-Escoda, Castro-Zubizarreta y FandosIgadod (2016d): si se analiza la realidad globalizada a la cual se enfrenta la 
Instituto Internacional de Investigación y Desarrollo Tecnológico Educativo INDTEC, C.A.

DOI: https://doi.org/10.29394/Scientific.issn.2542-2987.2020.5.16.15.290-304

OAI-PMH: http://www.indteca.com/ojs/index.php/Revista_Scientific/oai

Ensayo Original / Original Essay

sociedad, en cualquiera de sus esferas, salta a la vista la urgente necesidad de atender a dicho llamado, es por ello que los nuevos modelos educativos tienen que dar atención a una nueva competencia que es necesaria hoy en día, la competencia digital, siendo esta una capacidad fundamental que debe adquirir el estudiante como parte de su perfil de egreso.

Por su parte, Silva (2018b): contrariamente a lo que se ha expresado, y en un contexto local, expone que el modelo educativo ecuatoriano, hace referencia a un perfil de salida que se desarrolla mediante la implementación de destrezas con criterio de desempeño. Es decir, a manera de ejemplo, si se compara el modelo educativo español y el ecuatoriano, se puede observar que dista mucho de las capacidades de desarrollo que cada uno quiere lograr, el avance desarrollado en base a destrezas dificulta, aún más, el desarrollo de competencias digitales en los procesos educativos ecuatorianos.

Como lo señala el Ministerio de Educación, Cultura y Deporte (2015b), en el Boletín Oficial del Estado:

La competencia digital es aquella que implica el uso creativo, crítico y seguro de las tecnologías de la información y la comunicación para alcanzar los objetivos relacionados con el trabajo, la empleabilidad, el aprendizaje, el uso del tiempo libre, la inclusión y participación en la sociedad. Esta competencia supone, además de la adecuación a los cambios que introducen las nuevas tecnologías en la alfabetización, la lectura y la escritura, un conjunto nuevo de conocimientos, habilidades y actitudes necesarias hoy en día para ser competente en un entorno digital (pág. 6995).

Pese a toda la información que apuntan a la importancia de las competencias digitales, la realidad se aleja mucho de los anhelos, un estudio realizado por Pérez-Escoda, Castro-Zubizarreta y Fandos-Igado (2016e): con un enfoque cuantitativo que involucró a 678 estudiantes de Educación General Básica (EGB), entre los cuales el $52,4 \%$ son varones y el $47,6 \%$ son mujeres, y en edades que oscilan entre 7 a 12 años, evidenciaron resultados poco 
alentadores, solo el $5 \%$ de toda la muestra alcanzó un nivel superior y avanzado, frente al 22,5\% que presentaron ausencia total de competencias digitales. Entre las conclusiones, se enmarca que la mera convivencia, exposición y uso de la tecnología, no desarrolla competencias digitales, esto se contrapone a lo que se espera de la Generación Z, quienes precisan de una intencionalidad educativa, ya que no es suficiente el acceso o la utilización de recursos tecnológicos, sino acompañar estos procesos educativos con desarrollo de competencias digitales, primero del docente y posteriormente del estudiante.

En efecto, el desarrollo de competencias en estos nuevos ciudadanos puede ir más allá de la mera utilización de elementos tecnológicos, su desempeño puede llevarlos a ocupar nuevas profesiones e integrarse con mayor facilidad en este mundo globalizado. Desde la perspectiva de Vilanova y Ortega (2017a): los Z proponen organizar el trabajo de manera diferente, su punto de vista prioriza un ambiente laboral favorable y el bienestar familiar, se enfocan más en recursos virtuales que potencien el famoso teletrabajo, del que tanto se habla en estos días, su pensamiento se encamina a ideales nuevos quizá vistos como retos de un futuro no muy lejano.

Siguiendo a Camacho (2018b); y Vilanova-Ortega (2017b): esta nueva visión, sólo será efectiva si existe una mejora en el plano educativo, para esta nueva generación y las venideras, es necesaria la incorporación de la tecnología y recursos digitales que potencien la parte práctica de los estudiantes como una característica principal que poseen los $Z$, de lo contrario, no se estaría dando respuesta al nuevo mercado laboral que precisa la sociedad actual.

En este sentido se habla ya de la Educación 3.0, cuyo objetivo deja atrás la educación tradicionalista 1.0 y la educación constructivista 2.0, para centrarse en la conectivista con la incorporación de recursos digitales, así como se puede apreciar en la figura 1. 
Figura 1. Circulo Hermenéutico de Dilthey: Salto de la Educación 1.0 hacia la Educación 3.0.

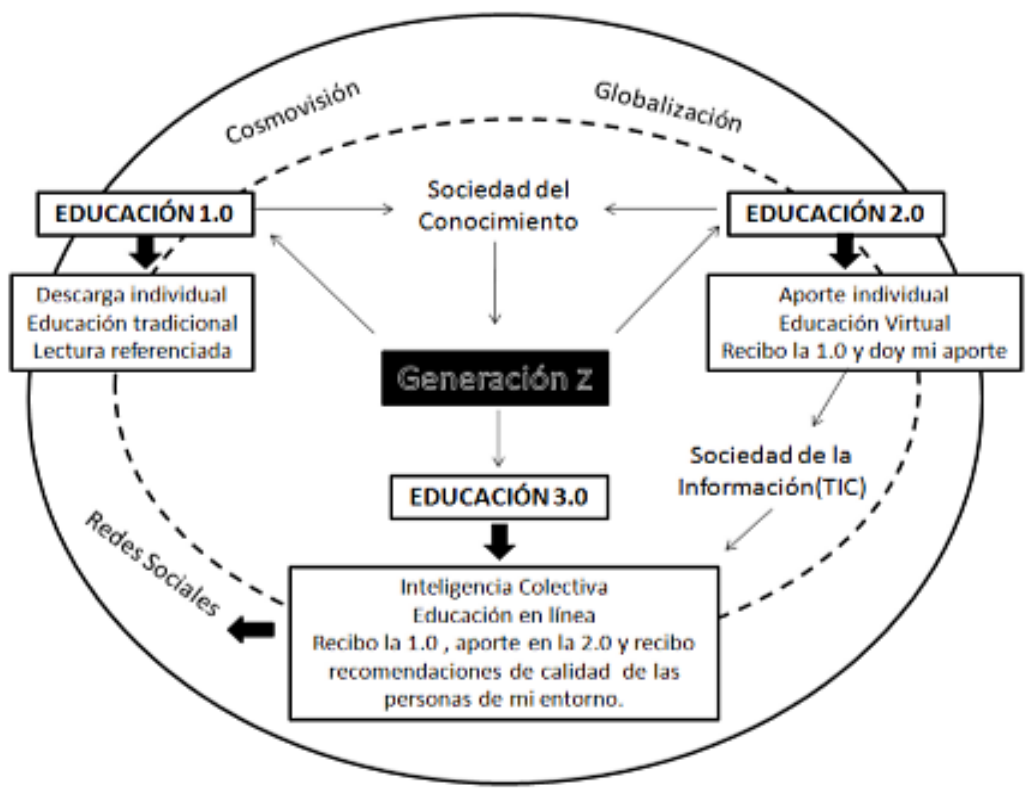

Fuente: Camacho (2018).

\section{Conclusiones}

La Generación Z, comprende un grupo generacional que ha nacido bajo la implementación de la tecnología como un recurso que forma parte de la cotidianidad, para estos nativos digitales la diversión, el ocio e incluso la formación, están inmersos en el uso de dispositivos como smartphones, tablets, laptops, entre otros. Dichos objetos no son meros inventos, son parte fundamental de su vida y mediante ellos se relacionan con la sociedad globalizada de hoy, la utilización de recursos de la web supone la visualización del mundo pues si algo no está allí simplemente no existe.

Contrariamente a la percepción aislada y pasiva que se tienen de esta generación, hay que considerar su preocupación por aspectos como la familia, la educación o la organización del trabajo. Tanto así, que consideran a la educación y el desarrollo de competencias digitales como las responsables de 
prepararlos para el desarrollo de nuevas profesiones que se enfoquen en el teletrabajo, priorizando el bienestar familiar y un buen ambiente laboral.

La construcción de conocimiento de la Generación Z y su transición hacia una educación conectivista denominada 3.0, debe enfocarse en el desarrollo de competencias digitales que puedan explotar su valioso potencial, caso contrario no existe mayor diferencia entre los inmigrantes digitales, es la intencionalidad y la educación virtual lo que supondrá una diferencia demostrativa en los niños y jóvenes que hoy están en proceso de formación y que han sido denominados Generación Z. Es difícil realizar aún predicciones a futuro, sin embargo, resulta muy interesante conocer cómo será el desempeño de estos nuevos ciudadanos que simbolizan un nuevo modelo de aprendizaje, de consumidores y seguramente de trabajadores.

Es necesario repensar el currículo ecuatoriano pues la realidad curricular maneja un enfoque basado en destrezas que, según Silva (2018c): buscan dar el salto hacia el desarrollo de competencias a futuro, todo ello pensando en una educación superior, sin embargo, esto presenta una falencia, ya que los estudiantes, independientemente de la educación que posean (fiscal, fisco-misional o privada), deben aprobar obligatoriamente el tronco común de materias, aquí radica la problemática, ya que las competencias necesitan más que conocimientos operativos, necesitan habilidades superiores de pensamiento que permitan al estudiante, desarrollarse en un mundo globalizado que dé respuestas a sus limitaciones. Si se analiza la universalidad tecnológica que se maneja hoy en día, es fundamental la generación de competencias digitales, pero lamentablemente el avance curricular del Ecuador, todavía no mantiene el enfoque óptimo para la consecución de estas capacidades.

La profesionalización docente es una de las preocupaciones más grandes para afrontar el desarrollo de competencias digitales en el estudiantado, misma que representa un reto al modelo educativo ecuatoriano, 
tanto su preparación inicial como posterior pueden generar un plus en el apoyo oportuno hacia una educación 3.0 que potencie la innovación y creatividad de la Generación Z. En busca del desarrollo de competencias, se contraponen dos realidades el docente y el estudiante, razón por la que el docente que no está capacitado a profundidad, no podrá desarrollar estas habilidades en sus estudiantes, no se puede preparar a ciudadanos del Siglo XXI con metodologías del Siglo XIX.

\section{Referencias}

Camacho, C. (2018a,b). Visión Teórica Humanística Educativa de la Generación Z 3.0 en Tiempos Complejos. Revista Scientific, 3(9), 2038, e-ISSN: 2542-2987. Recuperado de:

https://doi.org/10.29394/Scientific.issn.2542-2987.2018.3.9.1.20-38

Fernández-Cruz, F., \& Fernández-Díaz, M. (2016a,b,c). Los docentes de la Generación $\mathbf{Z}$ y sus competencias digitales. Revista Científica de Educomunicación, XXIV(46), 97-105, ISSN: 1134-3478; e-ISSN: 19883293. Recuperado de: https://doi.org/10.3916/C46-2016-10 Ministerio de Educación, Cultura y Deporte (2015a,b). Orden ECD/65/2015. Boletín Oficial del Estado, (25), 6986-7003, ISSN: 0212-033X. Madrid, España: Junta de Defensa Nacional del Ministerio de la presidencia, relaciones con las cortes y memoria democrática.

Ortega, I. (2017). Generación Z: El dilema. España: Deusto Business School. Pérez-Escoda, A., Castro-Zubizarreta, A., \& Fandos-Igado, M. (2016a,b,c,d,e). La competencia digital de la Generación Z: claves para su introducción curricular en la Educación Primaria. Revista Científica de Educomunicación, XXIV(49), 71-80, ISSN: 1134-3478; e-ISSN: 1988-3293. Recuperado de: https://doi.org/10.3916/C49-2016-07

Silva, J. (2018a,b,c). Educación Basada en Competencia (EBC) y los saberes para una educación del futuro, en Ecuador. Certiuni 
Instituto Internacional de Investigación y Desarrollo Tecnológico Educativo INDTEC, C.A.

DOI: https://doi.org/10.29394/Scientific.issn.2542-2987.2020.5.16.15.290-304

OAI-PMH: http://www.indteca.com/ojs/index.php/Revista_Scientific/oai

Ensayo Original / Original Essay

Journal, (4), 9-19, e-ISSN: 2444-5800. Recuperado de:

$\stackrel{\frac{0}{5}}{\frac{\pi}{N}}$ http://www.uajournals.com/certiunijournal/es/revistaes/numerosanterior es.html?id=141

Vilanova, N., \& Ortega, I. (2017a,b). Generación Z: Todo lo que necesitas saber sobre los jóvenes que han dejado viejos a los millennials. Primera edición, ISBN: 978-84-17114-31-2. Barcelona, España: Plataforma Editorial. 


\section{Ensayo Original / Original Essay}

\section{David Ismael Delgado Velesaca}

e-mail: david.delgadov@outlook.com

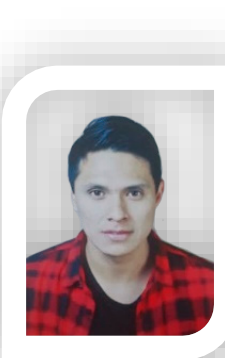

Nacido en la ciudad de Cuenca, Ecuador el 15 de febrero del año 1990: Realicé mis estudios secundarios en el colegio Nacional César Dávila Andrade; y mis estudios de tercer nivel en Universidad de Cuenca (UCUENCA), obteniendo el título de "Licenciado en Educación General Básica"; en este momento trabajo como docente en la Unidad Educativa Particular (CEDFI), de la ciudad de Cuenca, en sexto grado de educación general básica. 


\section{Diego Heriberto Urgilés Quintuña}

e-mail: diegourgilesq@hotmail.com

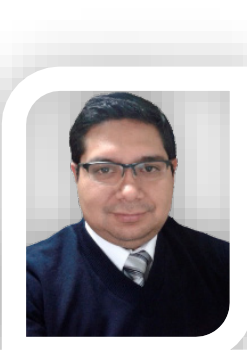

Nacido en la ciudad de Riobamba, Ecuador, el 9 de marzo del año 1981. Los estudios secundarios los realicé en el Colegio Militar No. 4 "Abdón Calderón" de la Ciudad de Cuenca; los estudios de tercer nivel en la Universidad Politécnica Salesiana (UPS), facultad de Ingeniería, el título obtenido fue de Ingeniero Electrónico; he ejercido trabajos a fin con título de tercer nivel; actualmente trabajo como profesional independiente en el área de Ingeniería Electrónica. 


\section{Patricia Katherine Vega Feijoó \\ e-mail: patricia.vegaf@outlook.es}

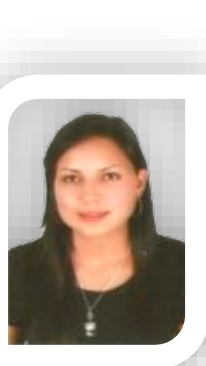

Nacida en el cantón Piñas, provincia de El Oro, Ecuador, el 7 de septiembre del año 1989. Realicé mis estudios secundarios, en España, en el Instituto de Educación Segundaria Domingo Valdivieso (IES); posteriormente obtuve el título de Técnico Superior en Administración y Finanzas; Mis estudios de pregrado los formalicé en la Universidad de Cuenca (UCUENCA), obteniendo el título de "Licenciada en Educación General Básica"; en este momento trabajo como docente en la Unidad Educativa Particular Alborada, de la ciudad de Cuenca, en quinto grado de educación general básica.

El contenido de este manuscrito se difunde bajo una Licencia de Creative Commons ReconocimientoNoComercial-Compartirlgual 4.0 Internacional 